\title{
Urinary excretion of calcium, magnesium, phosphate, citrate, oxalate, and uric acid by healthy schoolchildren using a 12-h collection protocol
}

\author{
Bernd Hoppe
}

Received: 13 June 2014 /Revised: 17 July 2014 / Accepted: 21 July 2014 / Published online: 31 August 2014

(C) IPNA 2014

\begin{abstract}
Although we do not have reliable data for the true prevalence of urolithiasis during childhood, the number of patients seen in outpatient clinics and admitted for stonerelated problems is steadily increasing worldwide. As for most pediatric patients a metabolic disease is the reason for stone development, because a high number of patients have severely recurrent urolithiasis, early and proper diagnostic evaluation is necessary to begin adequate and preventive treatment. However, diagnostic evaluation, especially in infants and younger children, is not always easy, and frequently a diagnosis is made late. Diagnostic evaluation should start with repeated urine analysis; but how and which urine should be collected and analyzed? What is the best and most accurate method for urine collection? In a paper published in a recent issue of Pediatric Nephrology, Torres and colleagues describe a more simplified method of urine collection. They propose analysis of late-afternoon spot-urine samples as well as an overnight collection of urine as the most appropriate to evaluate patientspecific urinary risk factors. Is this truly the case?
\end{abstract}

Keywords Renal transplantation $\cdot$ Pediatric $\cdot$ Renal mass

All children with a first kidney stone or the diagnosis of nephrocalcinosis should be screened for metabolic reasons for stone disease in order to start preventive measures as soon as possible $[1,2]$. However, what is the best way to collect adequate urine sample(s) first to gain normal age-related values, and secondly to obtain adequate diagnostic information? Only if the normative data depict a true "normal" and not

B. Hoppe $(\square)$

Division of Pediatric Nephrology, University Children's Hospital

Bonn, Adenauerallee 119, 53113 Bonn, Germany

e-mail: bernd.hoppe@UKB.uni-bonn.de an artificial situation of life, are they ready for use. This also applies to urine collected for diagnostic purposes. THE REAL LIFE risk factor for stone disease has to be evaluated - and this can sometimes be problematic!

There are many pitfalls to observe: how can we exclude "environmental" influences, like dietary excess or extreme fluid intake just at the time of collection? How can we avoid missed portions of a $24-\mathrm{h}$ urine sample in a small child? Is it reliable if diagnosis is made/excluded by one/some spot urine(s)? Would it always be better to ask for timed urine collections of any time frame (e.g., 4, 6, $12 \mathrm{~h}$ )? Is it practicable to then extrapolate each single parameter to a 24-h excretion and relate that to body surface area or body weight? Or is calculation of molar creatinine ratios better for interpreting a patient's specific risk factors? Is it sufficient to exclude a problematic diagnosis (e.g., a metabolic disorder) on the basis of just one urine collection? Is it recommendable to give dietary advice before the start of collection or to even force changes in nutrient intake at the time of urine sampling to gain further information on diet-related risk factors?

Obviously a lot of questions need to be answered which form the background of the paper by Sáez-Torres and colleagues in a recent issue of Pediatric Nephrology [3].

The main purpose of this paper was to establish normative excretion data based on a simplified collection protocol. Urine collections, especially in smaller children, are frequently problematic, so a simplified reliable sample method would be very much appreciated. For the authors, simplification meant analysis of lithogenic and stone inhibitory parameters in an afternoon spot urine collected $2 \mathrm{~h}$ after a light meal (influence of diet), but also in a 12-h overnight urine collection (no or less dietary influence). Urine from children with or without a family history of urolithiasis was examined and compared. It was agreeably concluded that for the purpose of finding normal age-related values, children with a family history of 
urolithiasis should be excluded in the process of achieving normative data. However, is their approach of urine collections truly practicable and recommendable?

I must admit I was puzzled by the idea of asking my patients to just collect a late afternoon spot urine sample and an overnight urine sample for analysis of lithogenic, here calcium, phosphate, oxalate and uric acid, and inhibitory parameters, here citrate and magnesium. Could this be the state of the art? First, I would like to focus on the late afternoon spot urine, which is taken $2 \mathrm{~h}$ after a light meal. Many times I have been surprised by the extreme (daily) fluctuation of molar creatinine ratios calculated from spot urine analysis. Apparently, these values are highly dependent upon fluid intake and diet, or differences in endogenous production rates (e.g., in primary hyperoxaluria) and of course urine creatinine concentration. Only if merely all spot urine samples, collected (over a number of days), are pointing in the same diagnostic direction, can we be sure about the reliability of our diagnostic approach. On the other hand, one or two outside values might still be suspicious for a severe kidney stone-related disease if the patient or family history leads towards such a diagnosis. For example, we would have missed diagnosis of primary hyperoxaluria type I in the sibling of an index patient by just relying on some spot urine values and the calculation of molar creatinine ratios, most of them in the normal range and only one being severely elevated [4]. Further diagnostic evaluation by repeated 24-h urine analysis and later by genetic examination led to diagnosis and treatment.

Clinical work in a huge pediatric kidney stone outpatient clinic definitively shows that it is possible to receive reliable urine samples - if the parents and the patients are adequately informed and trained. Our approach takes into account that we first would like to get an impression about the "normal" situation at home, but later also about dietary influences on urinary excretion parameters.

The first and important advice is not to increase the daily fluid intake dramatically ("just for the doctor"), when urine is collected for the analysis of lithogenic and stone inhibitory substances. Such an extreme increase in fluid intake, and hence urine volume, may mask the true patient-related risk factor. For example, many little girls have just a singular risk factor for stone disease: low fluid intake. If they are forced to drink as much as they can just at the time of collection(s), we may never find the reason for urolithiasis.

Although most of our pediatric patients may have a metabolic basis of kidney stone disease [1,2], we still have to also search for other factors leading to urolithiasis or nephrocalcinosis [5]. In this respect, it is truly important to take into consideration that the urine collected overnight may depict metabolic/genetic diseases, but will not give evidence for environmental factors influencing urinary excretion parameters, here most obviously nutrition. Such an influence will also not be excluded by late afternoon spot urine, early morning spot urine samples, or the analysis of a second urine voided in the morning (although frequently regarded as the most appropriate urine to use for urolithiasis-related analysis) $[6,7]$. I feel that the only correct approach would be to either collect spot urine at different times of 1 day, or even better on different days, or, much more preferably to collect (not only one) 24-h urine.

The parents of my patients always claim that it is extremely difficult to collect the night-time part of a 24-h urine. I most frequently receive the notice that one or two of the night voidings were missed. So, if we consider that a simplified urine collection method is necessary for diagnostic purposes, I would definitively not recommend a collection overnight.

What is my personal preferred procedure? I am interested in normal life situations, but also like to gain insight into "environmental" influences on urine excretion parameters. In infants and young children, repeated spot urine samples are taken at home. Parents have to give information on the time of collections, preferably we receive $2-3$ spot urine samples from different times of the day on repeated days. Also, we would like to get information on the normal dietary intake (specific normal values related to formula, or breast-fed infants [8]) and dietary excess (e.g., spinach, rhubarb) in the younger child [9]. As soon as 24-h urine collections are possible, I switch to that procedure, as, in my own experience, very frequently molar creatinine ratios of lithogenic and stone inhibitory factors or other calculated ratios (e.g., $\mathrm{Ca} / \mathrm{Ci}[10]$ ) fluctuate so much that a true diagnostic "red" line cannot be demonstrated.

For 24-h collections, parents and patients are advised that normal fluid intake is mandatory (no excess drinking at time of collections). Three 24-h urine samples are collected, one under "normal" diet, one under a lowoxalate diet, and one with a high-oxalate intake (spinach, rhubarb) and all stone risk factors are analyzed [2, 9]. In addition, a urinary $\mathrm{pH}$ profile is done depending on the first urine results (e.g., low citrate excretion) or stone analysis (e.g., phosphate stones). Only after urine collections give evidence for a specific risk factor further diagnostic evaluations are started. Hence, this reduces harm to the kids (e.g., less blood drawing) and enables us to convince insurance companies that such a procedure is economically meaningful (e.g., less expensive, risk factor-triggered diagnostic evaluation).

Nevertheless, the true gold standard of urine collection for the purpose of analysis of lithogenic and stone inhibitory parameters in the pediatric patient is still debatable. It is true that a simplified method would be very much appreciated, especially by the patients themselves (and the parents). However, my conclusion is that the presented procedures by SáezTorres et al. [3] still have too many pitfalls to be recommendable. The authors neither compared both the afternoon spot 
urine samples and also the overnight collection with 24-h urinary excretions, nor did the timed urine collection always correlate to the spot urine sample. Therefore, it remains unclear whether the data presented can reliably predict kidney stone risk in a given pediatric patient.

Very frequently, personal experiences are of prime importance regarding which procedure will be used. My diagnostic algorithm, with repeated 24-h urine collections still being the gold standard, will hence leave way for discussions.

\section{References}

1. Van't Hoff WG (2004) Aetiological factors in paediatric urolithiasis. Nephron Clin Pract 98:c45-c48

2. Hoppe B, Kemper M (2010) Diagnostic examination of the child with urolithiasis or nephrocalcinosis. Pediatr Nephrol 25:403-413

3. Sáez-Torres C, Rodrigo D, Grases F, García-Raja AM, Gómez C, Lumbreras J, Frontera G (2014) Urinary excretion of calcium, magnesium, phosphate, citrate, oxalate, and uric acid by healthy schoolchildren using a 12-h collection protocol. Pediatr Nephrol 29:1201-1208
4. Hoppe B, Danpure CJ, Rumsby G, Fryer P, Jennings PR, Blau N, Schubiger G, Neuhaus T, Leumann E (1997) A vertical (pseudodominant) pattern of inheritance in the autosomal recessive disease primary hyperoxaluria type 1: lack of relationship between genotype, enzymic phenotype, and disease severity. Am J Kidney Dis 29:36-44

5. Habbig S, Beck BB, Hoppe B (2011) Nephrocalcinosis and urolithiasis in children. Kidney Int 80:1278-1291

6. Hong YH, Dublin N, Razack AH, Mohd MA, Husain R (2010) Twenty-four-hour and spot urine metabolic evaluations: correlations versus agreements. Urology 75:1294-1298

7. Choi IS, Jung ES, Choi YE, Cho YK, Yang EM, Kim CJ (2013) Random urinary calcium/creatinine ratio for screening hypercalciuria in children with hematuria. Ann Lab Med 33: 401-405

8. Hoppe B, Roth B, Bauerfeld C, Langman CB (1998) Oxalate, citrate, and sulfate concentration in human milk compared with formula preparations: influence on urinary anion excretion. J Pediatr Gastroenterol Nutr 27:383-386

9. Hoppe B, Leumann E, von Unruh G, Laube N, Hesse A (2003) Diagnostic and therapeutic approaches in patients with secondary hyperoxaluria. Front Biosci 8:e437-e443

10. Sikora P, Zajaczkowska M, Hoppe B (2009) Assessment of crystallization risk formulas in pediatric calcium stone-formers. Pediatr Nephrol 24:1997-2003 